\title{
ЭКОЛОГИЯ
}

И ПРИРОДОПОЛЬЗОВАНИЕ

DOI: https://doi.org/10.15688/nsr.jvolsu.2019.2.4

UDC 330.4:316.422.44:330.34

LBC 65в 631

\section{QUALITY OF LIFE, USING SCIENCE AND ECONOMIC GROWTH AND MATHEMATICAL ANALOGUE MODEL OF THEIR INDICES}

\author{
Rem P. Zadneprovsky
}

Scientific Research Institute of Innovation Technologies, Volgograd, Russian Federation

\begin{abstract}
The aim of the work is to use the mathematical modeling apparatus to discover the relationship between real indicators of economic growth and well-being of the population with the quality of life of individual citizens. The subjective perception of the quality of life by the subject and the researcher makes this problem quite difficult for mathematical formalization. Digitalization of all types of human activity becomes one of the problematic aspects for modeling its status in the surrounding society. On the other hand, it allows you to Bank available solutions to the main tasks in human activity to create a comfortable living environment. The technique of formalization is considered and applied from the standpoint of General laws of motion and time dynamics of processes in natural environments (mechanical, electrical, biological systems), which may have a damping or exponential-wave character. Based on the proposed dependent equations, we offer a minimum list of factors that are necessary for the construction of predictive models. Taking into account the proposed factors, prognostic models are made that allow determining the current state of human quality of life with a sufficiently high probability in connection with the dynamic characteristics of the environment and socio-economic conditions of its life.

Key words: quality of life, scientific and technical forecast, reasonable consumption, social activity, innovation, analogue mathematical models.
\end{abstract}

УДК 330.4:316.422.44:330.34

ББК 65 в 631

\section{О КРИТЕРИЯХ КАЧЕСТВА ЖИЗНИ И ПРОГНОЗЕ ПОКАЗАТЕЛЕЙ НАУЧНО-ТЕХНИЧЕСКОГО И ЭКОНОМИЧЕСКОГО РАЗВИТИЯ С ИСПОЛЬЗОВАНИЕМ АНАЛОГОВЫХ МАТЕМАТИЧЕСКИХ МОДЕЛЕЙ}

\section{Рэм Петрович Заднепровский}

Национальный научно-исследовательский и проектный институт инновационных технологий, г. Волгоград, Российская Федерация

Аннотация. Рассматриваются вопросы повышения качества жизни населения, создания благоприятных условий для научно-прикладных исследований, разумного потребления, развития бизнеса и привлекательности инвестиций, основы научно-технического прогноза и примеры аналогового математического моделирования некоторых показателей социально-экономических процессов с учетом современных технологий управления бизнесом и социальных отношений.

Ключевые слова: качество жизни, научно-технический прогноз, разумное потребление, социальная активность, инновации, аналоговые математические модели. 
В настоящее время очевидна необходимость применения новых подходов к созданию благоприятных условий для развития научно-прикладных исследований, расширения сфер бизнеса и инвестиций. Важны целевые модели улучшения качества жизни с разработкой «дорожных карт» развития региона. Внедрение новых моделей способствует повышению деловой активности и комфортности среды обитания. Эти вопросы частично затронуты в ряде публикаций [1-12].

Прогноз населения земного шара к 2050 г. составит 9 млрд человек. Нас ждут технологические прорывы, новые мобильные устройства, искусственный интеллект, роботизация, автомобили-роботы, 3D-печать, нано- и биотехнологии, новое материаловедение, накопление и хранение энергии, квантовые вычисления. Революционные изменения распределены по основным трем блокам: физический (3D-печать, робототехника, новые материалы), цифровой (экономика совместного потребления - услуги по доставке, ремонту, выполнение административных заданий), биологический (генетика дает возможность варьировать те или иные биосвойства и устранять болезни). В центре развития и роста исследований и внедрений должны быть поставлены интересы населения регионов. Необходима разработка критериев разумного потребления и качества жизни.

Еще Гиппократ отметил, что «ни насыщение, ни голод и ничто другое нехорошо, если преступает меру природы».

Биологически обусловленный эгоизм, присущий всему живому, является одним из необходимых средств выживания. Для человеческих индивидуумов и отдельных групп и сообществ понятие эгоизм имеет более широкое значение. Можно говорить об эгоизме потребления как о неосознанном и осознанном стремлении к преимущественному потреблению всего, что не только удовлетворяет необходимые физиологические потребности, но и приносит удовольствие. Это может быть удовольствие от обладания редкой, дорогой вещью, от потребления недоступных большинству услуг, от размеров своей власти над другими, популярности, творческих результатов и т. п.
Научно-технические достижения (НТД) способствуют не только увеличению производства необходимых продуктов, но и товаров и услуг, являющихся нерациональными с точки зрения существования человечества, то есть таких, которые стимулируют эйфорию безудержного сверхпотребления (СП). При этом тип политического устройства государства может существенно влиять на ускорение и замедление СП.

СП ведет не только к очевидному увеличению экологического неблагополучия, но и к деградации общества, когда великий принцип гуманизма вытесняется индивидуальной свободой вседозволенности (СВ). Принцип СВ имеет тяготение к политическим устройствам типа олигархий, так как СВ принципиально не может быть доступна для всех.

Сейчас в мире стремительно формируются новые цифровые экономики [8]. На смену классическому государству приходит государство цифровое без четких границ, но со своими требованиями к бизнесу и гражданам. Цифровая экономика упрощает связь между людьми. Упраздняются посредники, так называемая экономическая надстройка бездельников.

Изучение платежеспособности, емкости рынка, новых разработок и технологий является основой для успешного развития управления инновационными проектами предприятий и методологии «новой экономики». В России показатель энергоемкости экономики в 2,53,5 раза выше, чем в развитых странах, что снижает конкурентоспособность Российской экономики. Одна из важнейших стратегических задач страны - сократить энергоемкость отечественной экономики к 2020 г. на $40 \%$. Нужны новые технико-экономические решения с оптимальным потреблением энергии всех видов и источников. Возможное снижение энергопотребления жилого дома площадью 10 тыс. м 2 до уровня 180 кВт/ч в год за 30 лет эксплуатации обеспечит экономию топлива и предотвращения выброса в атмосферу города 15 тыс. т $\mathrm{CO}_{2}$, что с точки зрения экологии эквивалентно 140 га леса. Качество жизни неразрывно связано с прогнозом научных достижений и разумностью потребления. В бытовом понимании термин качество определяет степень пригодности объекта для по- 


\section{ЭКОЛОГИЯ И ПРИРОДОПОЛЬЗОВАНИЕ}

требителя. В международном стандарте под качеством понимается совокупность характеристик объекта, относящихся к его способности удовлетворять установленные и предполагаемые потребности. При этом речь идет о наборе количественных и качественных требований к характеристикам объекта с возможностью их реализации и проверки. В социологии качество жизни (КЖ) выражается частными показателями удовлетворения материальных и культурных потребностей человека: качество питания, комфорта, окружающей среды, получаемых знаний и их развития и приложения к жизненным проблемам и ряд других. Индивидуальные потребности, формализованные индексами КЖ, нередко противоречат или мало соответствуют общественным нормам и неписанным правилам поведения. Индивидуальные психофизические особенности типов сообществ создают особые трудности для выбора и обоснования набора показателей (индексов). Существенную роль играет учет интереса (направленность субъекта на значимые объекты потребления) - как одного из комплексных критериев качества жизни. Социальный интерес можно рассматривать как импульс деятельности субъекта (личности), социального слоя, нации или общества. Оптимизация КЖ предполагает предварительный математический анализ с выделением социальных взаимодействий, наиболее общих стремлений и запросов индивидов и групповых сообществ, норм и правил, позволяющих участникам сообществ регулировать и прогнозировать социальное и научно-техническое взаимодействие. Любая математическая модель должна дополняться системой качественных показателей, например, в виде условных баллов относительного сопоставления, получаемых в результате статистического анализа опросов групп экспертов и тестирования отдельных групп населения $[1 ; 6$; $9 ; 13]$. Распределение качественных отличий количественных характеристик индивидуумов в больших выборках во многом аналогичны распределению частиц с данным уровнем их энергии или другого параметра их состояния. Существует невозможность полного переноса закономерностей взаимодействия двух индивидуумов (или частиц) на закономерности их коллективного взаимодействия. Для ис- пользования законов распределения в конкретных моделях (например, для прогноза потребления товаров и услуг) необходима их модификация с обоснованием краевых условий и ограничений. В ряде случаев может быть использовано предложенное автором выражение [5] для относительной доли множества $N$ с задаваемым критерием: $n=\mathrm{Nm}+(\mathrm{No}-$ $N m) \exp (q)$. Здесь $\mathrm{Nm}$ и $\mathrm{No}-$ верхний и нижний ограничительный уровень с присущим ему критерием, q-показатель, учитывающий относительный уровень, например, дохода - Д. Подобное выражение можно использовать для прогнозирования потребления товаров и услуг с различным качественным уровнем с оценкой вероятности распределения требований к объему таких услуг среди групп населения (потребителей) или (в пределе) среди индивидуумов), Такие критерии качества жизни как здоровье, уровень межличностного общения, возможность удовлетворения культурных запросов и индивидуальных потребностей в большей или меньшей степени зависят от величины Д. Важную роль в повышении общего показателя КЖ играет частный показатель $K$ повышение качества потребляемых товаров и услуг, культуры досуга и обслуживания. Количественная оценка этих показателей достаточно трудна. Соответственно, в материально-технической сфере это уровни: материалоемкости, трудоемкости, производительности, рентабельности, фондоемкости и энергоемкости. Подобно вышеизложенному, учитываются индексы финансовых возможностей, качества технологической системы, сбыта и качества конкурентоспособности продукции. Почти все частные коэффициенты определяются на основе статистических данных и вероятностно-аналитических экспертных оценок. Бурное развитие научно-технической специализации породило ряд проблем поддержания интеллектуального уровня достаточно высокого для востребования и широкого внедрения научных достижений (НТД). Глобальные задачи повышения КЖ и, в частности, экологические проблемы не могут быть решены только узкими специалистами. Назрела необходимость выработка критериев оптимального соотношения специалистов узкого профиля и энциклопедически образованных людей. Ближайшая задача - создание алго- 
ритмизированного информационного банка данных (доступных широкому кругу специалистов и населения) способная выдавать детальные решения в различных научно-технических и социально-экономических областях. Время требует создания освоения новых программ образования по проблемам адаптации к окружающей среде с учетом социально-экономических показателей, философии и социологии разумного потребления, научных основ оптимизации социально-политических решений. Ошибки в проведении государственной научно-технической политики неизбежно приводит к спаду экономики и КЖ. Отметим, что «голодное» финансирование, во многих случаях, приводит к недоброкачественности исследований и выводов и снижению качества специалистов. Рассмотрим некоторые общие соображения по выбору аналоговых математических уравнений. Процессы движения и длительного развития процессов в естественных средах (механических, электрических, биологических систем) носят затухающий или периодически возрастающий экспоненциальноволновой характер. Это касается всех естественных процессов природы и, несомненно, должно иметь аналоги в развивающихся обществах и, в частности, при изменении критериев КЖ. Отметим однотипность терминов развития процессов (или движения) в анализе естественно-научных и экономико-социальных процессов: импульс движения, движущая сила или потенциал, скорость и ускорение процессов, приращение (или спад) экономических или физических показателей. Все это может быть отражено в аналоговых дифференциальных уравнениях для различных развивающихся систем и процессов с привлечением различных вероятностно-статистических коэффициентов частных показателей. Такие явления, как деградация общества, инфляция, конкуренция, обесценивание знаний, падение КЖ, также могут найти аналоговое толкование, связанное с распространением энергии и переходом ее из одного состояния в другое. Рост КЖ трудно представить без роста качественных показателей общей культуры, включающей в себя и нравственно-воспитательный аспект, положительную (или отрицательную) значимость искусства. Наука, в значительной степени, является стратегическим компасом философии, призванной наметить разумные пределы дифференциации общества. На общественное сознание существенно воздействуют в глобальном масштабе: углубление кризиса современной цивилизации и возвышение роли ненаучного знания. Открытие тонких форм материи и информационного поля планеты выдвигает важную научно-естественную проблему о роли в развитии общества, так называемого «материального сознания». Известен философский постулат о переходе количества в качество. В математической модели это можно трактовать как определение меры количества массовой продукции (изготавливаемой в течении длительного времени, состоящего из многочисленных циклов) и длительности выпуска штучной продукции $-M$, по достижении которой, качество штучных изделий необходимо повышается. Величину $M$ следует выразить в безразмерном соотношении, например, в процентах от количественного множества $-n$. Необходимость повышения качества $-K$ связана величиной спроса на изделия (товары, услуги), насыщения сферы сбыта продукции, ростом квалификации изготовителей, внедрением инноваций НТД. Простейшее выражение для аналога качества: $K_{A}=\frac{n}{1+n} \kappa_{1} \kappa_{2 . . .} \kappa_{n}$ является своего рода параметром вероятностного ожидания небракованных (качественных) изделий. Так, например, при $n=1, K_{A}=0,5$; при $n=3$, $K_{A}=0,75$; при $n \rightarrow \infty, K_{A} \rightarrow 1$. Частные коэффициенты - к выражают отдельных циклов производства. Если число частных учитываемых количественных операций изготовления $\kappa \rightarrow \infty$, то величина $K_{A} \rightarrow 1 / e$, где $e=2,71-$ одно из так называемых «натуральных чисел», характеризующих естественных ход многих процессов. В данном случае можно полагать, что $K_{A}=M \approx 0,37$. Это число можно понимать как меру количественного перехода (37 \% п) в преимущественно качественный период. Если обозначить качество как соотношение $K=n_{\kappa} / N$, где п ${ }_{\kappa}-$ количество качественных изделий в конечном множестве $N$, то приращение коэффициента качества можно выразить в виде дифференциального соотношения: $d K=D\left(d n_{\kappa} / N\right)$, где $N=n_{\kappa}+n_{0}$ (сумме обычных и качественных изделий), $D$ - коэффициент, отражающий от- 
носительное влияние искусственных факторов влияющих на спрос (рекламная активность, недобросовестная конкуренция и др.). Научно- технический прогресс и прогнозирование его развития имеет большое значение для планирования и реализации крупных инвестиций в перспективных производствах товарной продукции. Основные методы прогноза основаны на: анализе развития принятых приоритетных отраслей, обеспечивающих, в первую очередь, нормальную жизнедеятельность человека; анализе и обобщения отраслевых публикаций научно-прикладных работ и изобретений с учетом статистики их реализаций (в России время реализации составляет 1025 лет), анализ диссертационных работ (наиболее обобщающие источники прогнозов); проведение экономического анализа динамики инвестиций в различные отрасли с учетом региональных данных; экспортных оценках отраслевого развития экономики с показателями качества жизни. В научных основах прогнозирования остается много нерешенных вопросов. К их числу относится выбор интервала времени, соотношения результатов прогноза, полученных по различным методам и группам анализа, мето-дикам оценки достоверности с учетом объема анализируемого материала и методам его ин-терпретации и многое другое. Стоит вопрос о возможности объективного научно-технического прогнозирования без учета политической обстановки и возможных социальных революций, политических переворотов с перео-риентацией социальных приоритетов, затяжных военных конфликтов, нередко связанных с амбициями групп людей и даже отдельных личностей и природных катаклизмов. На взгляд автора эти катаклизмы могут во многих случаях серьезно повлиять на выбор приоритетных отраслей развития и досто-верность результатов на прогнозируемый период. Процессы движения или развития поступательных процессов в природных средах и механических, электрических и биологических системах имеют, в большинстве случаев, затухающий или возрастающий характер с возможным колебательным изменением исследуемой характеристики). Состояние общества из множества субъектов (индивидов с личными свойствами и их групп) с постоянно сталкивающими интересами и про- тиворечиями и при наличии переменных воздействий природной среды и космоса не может быть адекватно описано системой реально решаемых уравнений. Однако отдельные достаточно автономные сообщества имеют более или менее развитые процессы саморегуляции своего развития и отдельные тенденции этого развития за достаточно длительный период-Т могут описаны достаточно простыми математическими уравнениями - аналогами уравнений физико-технических процессов. Рассмотрим основную цель этапного развития общества - повышение благосостояния его членов (субъектов). Обычно благосостояние оценивают величиной дохода - Д в реальных счетных единицах (рублях, долларах и др.). Реальный рубль и большинство других денежных единиц недостаточно устойчивы для учета Д, что связано с общей экономикосоциальной неустойчивостью и спекулятивностью многих вариантов денежного обращения. Чрезмерная изменчивость таких единиц мало пригодна для использования в прогнозных математических моделях. Можно использовать изменение цены продукции и услуг - Ц. Однако, теоретически, предпочтительнее использовать единицы энергии - Э (или работы). Затраты энергии на производство необходимых товаров отражают более объективно стоимость труда, конкурентоспособность, ценообразование. Можно считать, что аналог прироста дохода как прожиточного минимума $\ni_{M}=N e / t$, где $N$-число членов сообщества, $e$ - среднее количество энергии для допустимого (или достигнутого) уровня жизни. Величина $e / t$ может трактоваться и как заработная плата в энергетических единицах (Дж) за время $t$. Общая энергия Э $=Э_{м}+Э_{\partial}+Э н$, где Э ${ }_{\partial}-$ добавочная энергия, Э

Энтропия отражает невосполнимые потери энергии и входит в общие непроизводительные потери энергии близкие к расходам на амортизацию - износ материально-технических средств и поддержание здоровья уже неработающих членов общества. Двигательной силой социально-экономического прогресса (СЭП) является наиболее активная часть членов сообщества (лидеров разработки и внедрения НТД, организации производства). В больших сообществах (множества $-n$ ) число таких членов - вероятностная вели- 
чина, которая может быть приближенно выражена аналогом на основе формулы БозеЭнштейна в упрощенном виде: $n / N_{a}=\exp [(w-$ $\left.\left.w_{0}\right) / E\right]$, где $w$ - энергетический потенциал индивида, $w_{0}$ - осредненный показатель этого потенциала для общей массы производителей, $E$ - аналог кинетической энергии постоянной Больцмана, зависящей от температуры физической среды. Эта величина может трактоваться как некоторый запас энергии (в виде материально-финансовых активов) у соответствующей части общества. Если учесть, что движущей производительной силой (ДПС) общества является небольшая прослойка наиболее энергичных предприимчивых членов, а большая часть субъектов являются просто ПС (производительной силой), то благосостояние в виде дохода распределяется крайне неравномерно. Отметим важное значение таких факторов как производство невостребованных товаров, сверхпотребление, потери энергии вследствие неразумного использования, недостаточно используемых научно-технических достижений (НТД) и аналога энтропии энергии - eе безвозвратных непроизводительных потерь. Величина дохода отражает совокупность финансово-материальных активов отдельного субъекта или всего общества. Простейшее дифференциальное аналоговое уравнение изменения дохода за некоторый период:

$$
Д=Д_{0} \pm B d Д / d t=Д_{0} \pm B Д^{*}
$$

Здесь Д* - приращение дохода (его дифференциал), $B$ - коэффициент, отражающий совокупность влияния социально-экономических условий. При изменении величины Д от $Д_{0}$ до $Д_{m}$ (максимума или минимума дохода), общее решение уравнения (1) имеет вид:

$$
\not=Д_{m}-\left(Д_{m}+Д_{0}\right) e^{-K t / B}
$$

Величина коэффициента $B$ можно трактовать как время - $T$ прогнозного цикла (планового изменения Д). Величина коэффициента $K$ в первом приближении может быть представлена в виде $K=\left(k_{1} k_{2} \ldots k_{3}\right)^{1 / i}$,где коэффициенты $k_{1 . . .} k_{i}$ учитывают отдельные (числом $i$ ), относительные вероятностные показатели производства и экономического состоя- ния (объема и качества продукции, конкурентоспособности, социально-политической стабильности, использования НТД, энергетические затраты на производство и поддержание принятого уровня качества жизни (КЖ), и др.). В более детальных анализах учитывается состояние рынков сбыта, финансовых возможностей, мощности производства, материально-технического обеспечения, уровень организации труда, компетенция кадров, информационных возможностей. Коэффициент $0<k<1$, имеет вероятностно-статистический характер и может быть оценен группой экспертов. Снижение коэффициента фактически удлиняет время цикла экономических преобразований. Другим простым примером аналогии может служить соотношение, вытекающее из уравнения Циолковского (разгон ракеты с некоторым запасом топлива (как аналога материально-финансового актива). Дифференциальное соотношение приращения основного актива для улучшения КЖ пропорционально изменению дохода и имеет вид:

$$
d m / m=-k d D / D_{0}
$$

После интегрирования этого уравнения получаем:

$$
m / m_{0}=k \exp \left(-D / D_{0}\right)
$$

Это уравнение показывает замедление роста дохода $\mathrm{D}$ предприятий или сообщества с начальным невосполняемым объемом активов $-m_{0}$. Здесь $m$ и $k$ соответственно - размер активов при данном доходе и коэффициент коррекции близкий по смыслу коэффициенту $K$. Из (3a) следует, что доход (или прибыль) пропорционален логарифму $-\ln \left(m_{0} / m\right)$. Развитие процессов общества с ограниченными внешними связями при отсутствии внешних импульсов энергии развития (займы, неравноправные договора о экономическом сотрудничестве и т. п.) во многих случаях имеет аналогии с автоколебательным движением каких-либо природных или технических систем под действием некоторого внутреннего импульса. Автоколебательные системы (AC) характеризуются: способностью генерировать собственные колебания, образуя их за счет поступательного движения (начального толчка); наличием собственного источника 


\section{ЭКОЛОГИЯ И ПРИРОДОПОЛЬЗОВАНИЕ}

энергии и системы ее распределения (например системы клапанов); обратной связи (влияние колеблющейся системы на работу клапанов (рычагов) управления) - аналога демократического управления обществом. Самовозбуждение $\mathrm{AC}$ из состояния покоя (плавное или жесткое) - от толчка. В последнем случае система более устойчивая, ее труднее вывести из равновесия. В АС переменная сила обратной связи должна совершать работу, то есть как аналог положительного сдвига в использовании энергии толчка для повышения качества жизни. При этом работа совершается, если фазы движущей периодической силы и скорости изменения положительного фактора (например аналога дохода) совпадают по фазе или сдвинуты на $90^{\circ}$. Важно отметить, что при гармоничном движении работой высших гармоник периодических сил часто можно пренебречь. Это позволяет использовать в качестве аналоговых математических моделей социально-экономических процессов линейные однородные уравнения типа:

$$
A x^{* *}+B x^{*}+C x=\text { const }
$$

Здесь $x^{* *}$ и $x^{*}$ - вторая и первая производные функции параметра - аналога от времени $x(t)$, а коэффициенты $A, B, C$ - соответствующие аналоги сил сопротивления движению (развитию процесса повышения доходов, изменения энергетического потенциала общества и других процессов, например, накопления капитала). Следует заметить, что правая часть уравнения (4) может быть переменной величиной. Отметим, что общие свойства физической АС отвечают базисному принципу автономии экономики - способности саморегуляции на основе внутрихозяйственных связей. Коэффициенты $B$ и $C$ сопротивления движению в уравнении - аналоге имеют также смысл некоторых сопротивлений движению (развитию) и ее движущей силы. Если величина $x=Э$, то в аналоговой модели положительным сопротивлением можно считать потребителя энергии (в условных единицах), а отрицательным сопротивлением - отбор энергии от системы (при противофазном смещении движущей силы и скорости аналогового процесса). Реальные колебательные процессы сопровождаются внутренней диссипацией энергии при наличии неравномерности развития как физического, так и экономического процесса. Эти релаксационные колебания имеют разрывной, пикообразный характер. Амплитуда таких колебаний почти не зависит от нагрузки. В физическом уравнении (4) коэффициенты $A, B, C$ определяются массой и сопротивлением движению (вязкой и упругой составляющей сил сопротивления, зависящих от скорости и перемещения). Для аналогового уравнения указанного типа можно использовать такие термины как масса производимых товаров, производительность, стоимость - цена (в реальных или условных единицах), ускорение дохода или прибыли, соотношения реализации (сбыта) товаров и услуг, накопление капитала и ряд коэффициентованалогов сопротивлений экономическому развитию, учитывающих трудности процесса развития (как правило экспертного вероятностно-оценочного характера с учетом статистического анализа). Рассмотрим пример аналогового уравнения, имеющего вид:

$$
a \Pi^{* *}+{ }_{b} \Pi^{*}+c \Pi=E
$$

Для автономного сообщества (при отсутствии существенных внешних импульсов развития $-E)$ : $\Pi^{* *}+8 \Pi^{*}+c \Pi=0$. Здесь $B=8 / a$, $C=c / a, \Pi-$ экономический эквивалент ценности (эи) произведенной продукции, необходимой для достижения некоторого уровня качества жизни (КЖ). Единицей этого эквивалента может служить твердая валюта, золото или другая условная единица, например, количества труда (в энергетических единицах). Тогда величины $и$ мпульсов процесса развития экономики $\Pi^{*}$ и $\Pi^{* *}$ определяются как скорость и ускорение их нарастания (или спада) эи/t и эи/ $t^{2}$, где $t$ - промежуток времени. Величина $\Pi^{* *}=m^{* *} Ц$, где $m^{*}$ соответствует производительности труда (выпуска массы товаров в единицу времени), а $m^{* *}$ - соответствующее ускорение и $Ц-$ условная цена единицы продукции. Коэффициент $B$, как аналог «вязкого» сопротивления, отражает рентабельность производства (интенсивность сбыта) и факторы, препятствующие сбыту продукции $\left(m_{c}\right)$, выражается в виде: $B=k_{c}\left(m_{c}{ }^{*} / m\right)$. Здесь $\kappa_{c}$ - коэффициент, учитывающий потери части возможной прибыли за счет необходимых 
расходов, связанных с ускорением реализации товарной массы. При детальном анализе этот коэффициент также, как отмеченный выше коэффициент $K$, определяется по произведению частных коэффициентов, учитывающих отдельные условия и формы сбыта. Рассмотрение частных значений коэффициентов является отдельной, и достаточно сложной задачей. Коэффициент $C$ отражает скорость накопления уровня неликвида $-\left(m-m_{c}\right) / m$ за время $t_{c}$ в виде:

$$
C=k_{n}\left[\left(m-m_{c}\right) / t_{c} m\right]\left[\left(Ц-Ц_{s}\right) / Ц t_{c}\right]
$$

Это произведение аналога скорости накопления неликвида и скоростного изменения ценового уровня, где $Ц$ и $\bigsqcup_{H}$ - соответственно, цена быстро реализованной продукции и демпинговая цена неликвида (в пределах расчетного периода). По аналогии с физическим уравнением частота колебаний скорости процесса $p=C^{1 / 2}$, а период гармонических колебаний $T=2 \pi C^{-1 / 2}$. Чем больше уровни сбыта и цены товаров, тем меньше частота и больше период данного этапа экономического развития и, соответственно, более устойчивый характер развития экономических процессов. Величина безразмерных коэффициентов $\kappa_{n}$ и $c$ колеблется между нулем и единицей. Решение уравнения (5) для автономного сообщества:

$$
\Pi=c_{1}\left[\exp \left(-\alpha_{1}\right)\right]+c_{2}\left[\exp \left(-\alpha_{2}\right)\right.
$$

где $\alpha_{1,2}=-0,5 B \pm\left(0,25 B^{2}-C\right)^{0,5}$.

Для выбора адекватного варианта следует рассмотреть частные случаи:

1) $B^{2} / 4=C, \Pi=\Pi_{0} \exp (-B t / 2), \alpha_{1}=$ $\alpha_{2}=B / 2$;

2) $B^{2} / 4>C$ (непроизводительные потери и сопротивление сбыту велики, возможны форс-мажорные обстоятельства и решение в форме (7) с постоянным спадом экономики);

3) наиболее важный случай: при условии, когда аналоговые силы сопротивления роста прибыли и, соответственно, развитию данного экономического этапа относительно малы.

Тогда решение уравнения имеет вид:

$$
\Pi=\Pi_{0}[\exp (-\alpha)] \sin \left(C^{0,5} t+\beta\right)
$$

Здесь коэффициент $\beta$ - угол сдвига фаз, указывающий на несовпадение амплитудных значений темпов импульсов производства (производительных сил) и скорости сбыта (реализации продукции). Формула (8) указывает на колебательный процесс с постепенно затухающими амплитудами колебаний, отражающими постепенное чередование прибыли-убытка с общим снижением величины П. Таким образом, рассмотренные аналоговые уравнения позволяют приближенно прогнозировать характер экономического развития и влияние на него отдельных факторов технико-экономического и социального значения.

\section{СПИСОК ЛИТЕРАТУРЫ}

1. Гун, Г. С. Управление качеством в метизном производстве / Г. С. Гун, М. В. Чукин // Металлургические процессы и оборудование. - 2013. № 4. - С. 106-112.

2. Борисоглебская, Л. Н. Моделирование коммерческой деятельности предприятий в условиях инновационных предложений / Л. Н. Борисоглебская, И. А. Миронова, С. М. Сергеев // Инновации. 2013. - № 1. - С. 8.

3. Вольфсон, Б. Л. Гибкое управление проектами и продуктами / Б. Л. Вольфсон. - СПб. : Питер, 2014. - $144 \mathrm{c}$.

4. Голубенко, О. А. Особенности разработки и внедрения менеджмента качества в испытательной лаборатории / О. А. Голубенко, А. А. Дедук // Евразийский союз ученых. - 2014. - № 7. - С. 485.

5. Заднепровский, Р. П. О математическом моделировании развития потенциала социально-экономических процессов / Р. П. Заднепровский // Актуальные вопросы экономики, менеджмента и финансов в современных условиях : сб. тр. 3-й Междунар. конф. - СПб. : ИЦРОН, 2016. -С. 152-156.

6. Евсеева, С. А. Проблемы несогласованности интересов субъектов хозяйствования в системе мененджмента организаций ЖКХ / С. А. Евсеева // Проблемы современной экономики. - 2012. № 4. - C. 299-303.

7. Ивантер, В. В. Экономический рост и экономическая политика в России / В. В. Ивантер, Б. Н. Порфирьев, А. А. Широв // ЭКО. -2014. - № 2. C. 55-67.

8. Кондрашов, Г. М. Ключевые факторы устойчивого развития - эффективность и инновационность / Г. М. Кондрашов, В. Б. Селезнев // Научные труды клуба докторов наук. - Волгоград : Сфеpa, 2017.- C. 31-38. 


\section{ЭКОЛОГИЯ И ПРИРОДОПОЛЬЗОВАНИЕ}

9. Мартыненко, Ю. В. Построение показателей операционной эффективности на основе теории массового обслуживания / Ю. В. Мартыненко // Современная экономика. Проблемы и решения. 2014. - T. 12, № 60. - С.18-26.

10. Масино, Н. Н. Стратегическая карта инновационного развития как инструмент интеллектуальной системы стратегического управления / Н. Н. Масино, М. А. Растов // Вестник Омского университета. Серия «Экономика». - 2015. - № 2. C. $159-162$.

11. Семенова-Слабкович, Я. А. Анализ подходов к исследованию теории поведения потребителя / Я. А. Семенова-Слабкович // Вестник Самарского госуниверситета. - 2013. - Т. 8, № 196. C. $14-18$.

12. Суслов, Е. Ю. Формирование управленческого знания устойчивого развития социальноэкономических систем : монография / Е. Ю. Суслов. -СПб. : СПБГИЭУ, 2012. - 256 с.

13. Patutina, E. S. The sysnem study of the functioningand motivation of staff for effective monitoring of quality activities for example autist branch of OOO «Gazprom transgaz samara» / E. S. Patutina, A. V. Raevskaya // General question of world science. - 2018. - P. 59-6414.

\section{REFERENCES}

1. Gun G.S., Chukin M.V. Upravlenie kachestvom $\mathrm{v}$ metiznom proizvodstve [Quality Management in Hardware Production]. Metallurgicheskie protsessy $i$ oborudovanie [Metallurgical Processes and Equipment], 2013, no. 4, pp. 106-112.

2. Borisoglebskaya L.N., Mironova I.A., Sergeev S.M. Modelirovanie kommercheskoy deyatelnosti predpriyatiy $\mathrm{v}$ usloviyakh innovatsionnykh predlozheniy [Modeling of Commercial Activities of Enterprises in Conditions of Innovative Proposals in the Future]. Innovatsii [Innovations], 2013, no. 1, p. 8.

3. Wolfson B.L. Gibkoe upravlenie proektami i produktami [Flexible Project and Product Management]. Saint Petersburg, Piter Publ., 2014. 144 p.

4. Golubenko O.A., Deduk A.A. Osobennosti razrabotki i vnedreniya menedzhmenta kachestva $\mathrm{V}$ ispytatelnoy laboratorii [Features of Development and Implementation of Quality Management in the Testing Laboratory]. Evraziyskiy soyuz uchenykh [Eurasian Union of Scientists], 2014, no. 7, p. 485.

5. Zadneprovsky R.P. O matematicheskom modelirovanii razvitiya potentsiala sotsialnoekonomicheskikh protsessov [On Mathematical Modeling of Potential Development of Social and Economic Processes]. Aktualnye voprosy ekonomiki, menedzhmenta $i$ finansov $v$ sovremennykh usloviyakh: sbornik trudov 3-y mezhdunarodnoy konferentsii [Current Issues of Economics, Management and Finance in Modern Conditions. Proc. $3^{\text {rd }}$ Intern. Conf]. Saint Petersburg, ITsRON Publ., 2016, pp. 152-156.

6. Evseeva S.A. Problemy nesoglasovannosti interesov subyektov khozyaystvovaniya $\mathrm{v}$ sisteme menendzhmenta organizatsiy ZhKKh [Inconsistency of Interests of Business Entities in the System of Management of Housing and Communal Services Organizations]. Problemy Sovremennoy Ekonomiki [Problems of Modern Economy], 2012, no. 4, pp. 299-303.

7. Ivanter V.V., Porfiryev B.N., Shirov A.A. Ekonomicheskiy rost i ekonomicheskaya politika v Rossii [Economic Growth and Economic Policy in Russia]. EKO [ECO], 2014, no. 2, pp. 55-67.

8. Kondrashev G.M., Seleznev V.B. Klyuchevye faktory ustoychivogo razvitiya - effektivnost i innovatsionnst [Key Factors of Sustainable Development: Efficiency and Innovation]. Nauchnye trudy kluba doktorov nauk [Scientific Works of Doctor Sciences Club]. Volgograd, Sphera Publ., 2017, pp. 31-38.

9. Martynenko Yu.V. Postroenie pokazateley operatsionnoy effektivnosti na osnove teorii massovogo obsluzhivaniya [Construction of Indicators of Operational Efficiency Based on the Theory of Queuing]. Sovremennaya ekonomika. Problemy i resheniya [Modern Economy. Problems and Solutions], 2014, vol. 12, no. 60, pp. 18-26.

10. Masino N.N., Rastov M.A. Strategicheskaya karta innovatsionnogo razvitiya kak instrument intellektualnoy sistemy strategicheskogo upravleniya [Strategic Map of Innovative Development As a Tool of Intellectual System of Strategic Management]. Vestnik Omskogo universtiteta. Seriya «Ekonomika» [Bulletin of the Omsk University. Series "Economy"], 2015, no. 2, pp. 159-162.

11. Semenova-Slabkovich Ya.A. Analiz podkhodov $\mathrm{k}$ issledovaniyu teorii povedeniya potrebitelya [Analysis of Approaches to the Study of Consumer Behavior Theory]. Vestnik Samarskogo gosuniversiteta [Bulletin of the Samara State University], 2013, vol. 8, no. 196, pp. 14-18.

12. Suslov E.Yu. Formirovanie upravlencheskogo znaniya ustoychivogo razvitiya sotsialnoekonomicheskikh sistem : monografiya [Formation of Managerial Knowledge in Sustainable Development of Socio-Economic Systems. Monograph]. Saint Petersburg, SPBSIEU Publ., 2012.256 p.

13. Patutina E.S., Raevskaya A.V. The System Study of the Functioning and Motivation of Staff for Effective Monitoring of Quality Activities for Example Autist-Branch ofOOO "Gazprom Transgaz Samara". General Question of World Science, 2018. pp. 59-6414. 


\section{Information about the Author}

Rem P. Zadneprovsky, Doctor of Sciences (Technology), Professor, Scientific Research Institute of Innovation Technologies, 13-y Gvardeyskoy St., 10, 400005 Volgograd, Russian Federation, nuput@mail.ru.

\section{Информация об авторе}

Рэм Петрович Заднепровский, доктор технических наук, профессор, Национальный научно-исследовательский и проектный институт инновационных технологий, ул. 13-й Гвардейской, 10, 400005 г. Волгоград, Российская Федерация, nuput@mail.ru. 